\title{
On the Continuity of Some Functions
}

\author{
Artur Korniłowicz \\ Institute of Informatics \\ University of Białystok \\ Sosnowa 64, 15-887 Białystok, Poland
}

Summary. We prove that basic arithmetic operations preserve continuity of functions.

MML identifier: TOPREALC, version: $\underline{7.11 .07 \quad 4.156 .1112}$

The terminology and notation used here have been introduced in the following articles: [20], [1], [6], [13], [4], [7], [19], [8], [9], [5], [21], [2], [3], [10], [18], [25], $[26],[23],[12],[22],[24],[14],[16],[17],[15]$, and [11].

\section{Preliminaries}

For simplicity, we adopt the following rules: $x, X$ are sets, $i, n, m$ are natural numbers, $r, s$ are real numbers, $c, c_{1}, c_{2}, d$ are complex numbers, $f, g$ are complex-valued functions, $g_{1}$ is an $n$-element complex-valued finite sequence, $f_{1}$ is an $n$-element real-valued finite sequence, $T$ is a non empty topological space, and $p$ is an element of $\mathcal{E}_{\mathrm{T}}^{n}$.

Let $R$ be a binary relation and let $X$ be an empty set. Observe that $R^{\circ} X$ is empty and $R^{-1}(X)$ is empty.

Let $A$ be an empty set. Observe that every element of $A$ is empty.

We now state the proposition

(1) For every trivial set $X$ and for every set $Y$ such that $X \approx Y$ holds $Y$ is trivial.

Let $r$ be a real number. Observe that $r^{2}$ is non negative.

Let $r$ be a positive real number. Note that $r^{2}$ is positive.

Let us note that $\sqrt{0}$ is zero. 
Let $f$ be an empty set. Note that ${ }^{2} f$ is empty and $|f|$ is zero.

The following propositions are true:

(2) $f\left(c_{1}+c_{2}\right)=f c_{1}+f c_{2}$.

(3) $f\left(c_{1}-c_{2}\right)=f c_{1}-f c_{2}$.

(4) $f / c+g / c=(f+g) / c$.

(5) $f / c-g / c=(f-g) / c$.

(6) If $c_{1} \neq 0$ and $c_{2} \neq 0$, then $f / c_{1}-g / c_{2}=\left(f c_{2}-g c_{1}\right) /\left(c_{1} \cdot c_{2}\right)$.

(7) If $c \neq 0$, then $f / c-g=(f-c g) / c$.

(8) $(c-d) f=c f-d f$.

(9) $(f-g)^{2}=(g-f)^{2}$.

(10) $(f / c)^{2}=f^{2} / c^{2}$.

(11) $|n \mapsto r-n \mapsto s|=\sqrt{n} \cdot|r-s|$.

Let us consider $f, x, c$. Observe that $f+\cdot(x, c)$ is complex-valued.

We now state a number of propositions:

$(\langle\underbrace{0, \ldots, 0}_{n}\rangle+\cdot(x, c))^{2}=\langle\underbrace{0, \ldots, 0}_{n}\rangle+\cdot\left(x, c^{2}\right)$.

(13) If $x \in \operatorname{Seg} n$, then $|\langle\underbrace{0, \ldots, 0}_{n}\rangle+\cdot(x, r)|=|r|$.

(14) $0_{\mathcal{E}_{\mathrm{T}}^{n}}+\cdot(x, 0)=0_{\mathcal{E}_{\mathrm{T}}^{n}}$.

(15) $f_{1} \bullet\left(0_{\mathcal{E}_{\mathrm{T}}^{n}}+\cdot(x, r)\right)=0_{\mathcal{E}_{\mathrm{T}}^{n}}+\cdot\left(x, f_{1}(x) \cdot r\right)$.

(16) $\left|\left(f_{1}, 0_{\mathcal{E}_{\mathrm{T}}^{n}}+\cdot(x, r)\right)\right|=f_{1}(x) \cdot r$.

(17) $\left(g_{1}+\cdot(i, c)\right)-g_{1}=\langle\underbrace{0, \ldots, 0}_{n}\rangle+\cdot\left(i, c-g_{1}(i)\right)$.

(18) $|\langle r\rangle|=|r|$.

(19) Every real-valued finite sequence is a finite sequence of elements of $\mathbb{R}$.

(20) For every real-valued finite sequence $f$ such that $|f| \neq 0$ there exists a natural number $i$ such that $i \in \operatorname{dom} f$ and $f(i) \neq 0$.

(21) For every real-valued finite sequence $f$ holds $\left|\sum f\right| \leq \sum|f|$.

(22) Let $A$ be a non empty 1-sorted structure, $B$ be a trivial non empty 1sorted structure, $t$ be a point of $B$, and $f$ be a function from $A$ into $B$. Then $f=A \longmapsto t$.

Let $n$ be a non zero natural number, let $i$ be an element of $\operatorname{Seg} n$, and let $T$ be a real-membered non empty topological space. Note that $\operatorname{proj}(\operatorname{Seg} n \longmapsto T, i)$ is real-valued.

Let us consider $n$, let $p$ be an element of $\mathcal{R}^{n}$, and let us consider $r$. Then $p / r$ is an element of $\mathcal{R}^{n}$.

One can prove the following proposition

(23) For all points $p, q$ of $\mathcal{E}_{\mathrm{T}}^{m}$ holds $p \in \operatorname{Ball}(q, r)$ iff $-p \in \operatorname{Ball}(-q, r)$. 
Let $S$ be a 1-sorted structure. We say that $S$ is complex-functions-membered if and only if:

(Def. 1) The carrier of $S$ is complex-functions-membered.

We say that $S$ is real-functions-membered if and only if:

(Def. 2) The carrier of $S$ is real-functions-membered.

Let us consider $n$. One can verify that $\mathcal{E}_{\mathrm{T}}^{n}$ is real-functions-membered.

Let us observe that $\mathcal{E}_{\mathrm{T}}^{0}$ is real-membered.

One can check that $\mathcal{E}_{\mathrm{T}}^{0}$ is trivial.

Let us observe that every 1-sorted structure which is real-functionsmembered is also complex-functions-membered.

Let us mention that there exists a 1-sorted structure which is strict, non empty, and real-functions-membered.

Let $S$ be a complex-functions-membered 1-sorted structure. One can check that the carrier of $S$ is complex-functions-membered.

Let $S$ be a real-functions-membered 1-sorted structure. Note that the carrier of $S$ is real-functions-membered.

Let us observe that there exists a topological space which is strict, non empty, and real-functions-membered.

Let $S$ be a complex-functions-membered topological space. Observe that every subspace of $S$ is complex-functions-membered.

Let $S$ be a real-functions-membered topological space. One can verify that every subspace of $S$ is real-functions-membered.

Let $X$ be a complex-functions-membered set. The functor $(-) X$ yields a complex-functions-membered set and is defined as follows:

(Def. 3) For every complex-valued function $f$ holds $-f \in(-) X$ iff $f \in X$.

Let us observe that the functor $(-) X$ is involutive.

Let $X$ be an empty set. One can verify that $(-) X$ is empty.

Let $X$ be a non empty complex-functions-membered set. Observe that $(-) X$ is non empty.

The following proposition is true

(24) Let $X$ be a complex-functions-membered set and $f$ be a complex-valued function. Then $-f \in X$ if and only if $f \in(-) X$.

Let $X$ be a real-functions-membered set. One can verify that $(-) X$ is realfunctions-membered.

Next we state the proposition

(25) For every subset $X$ of $\mathcal{E}_{\mathrm{T}}^{n}$ holds $-X=(-) X$.

Let us consider $n$ and let $X$ be a subset of $\mathcal{E}_{\mathrm{T}}^{n}$. Then $(-) X$ is a subset of $\mathcal{E}_{\mathrm{T}}^{n}$.

Let us consider $n$ and let $X$ be an open subset of $\mathcal{E}_{\mathrm{T}}^{n}$. Observe that $(-) X$ is open.

Let us consider $n, p, x$. Then $p(x)$ is an element of $\mathbb{R}$. 
Let $R, S, T$ be non empty topological spaces, let $f$ be a function from $R \times$ $S$ into $T$, and let $x$ be a point of $R \times S$. Then $f(x)$ is a point of $T$.

Let $R, S, T$ be non empty topological spaces, let $f$ be a function from $R \times$ $S$ into $T$, let $r$ be a point of $R$, and let $s$ be a point of $S$. Then $f(r, s)$ is a point of $T$.

Let us consider $n, p, r$. Then $p+r$ is a point of $\mathcal{E}_{\mathrm{T}}^{n}$.

Let us consider $n, p, r$. Then $p-r$ is a point of $\mathcal{E}_{\mathrm{T}}^{n}$.

Let us consider $n, p, r$. Then $p r$ is a point of $\mathcal{E}_{\mathrm{T}}^{n}$.

Let us consider $n, p, r$. Then $p / r$ is a point of $\mathcal{E}_{\mathrm{T}}^{n}$.

Let us consider $n$ and let $p_{1}, p_{2}$ be points of $\mathcal{E}_{\mathrm{T}}^{n}$. Then $p_{1} p_{2}$ is a point of $\mathcal{E}_{\mathrm{T}}^{n}$. Let us note that the functor $p_{1} p_{2}$ is commutative.

Let us consider $n$ and let $p$ be a point of $\mathcal{E}_{\mathrm{T}}^{n}$. Then ${ }^{2} p$ is a point of $\mathcal{E}_{\mathrm{T}}^{n}$.

Let us consider $n$ and let $p_{1}, p_{2}$ be points of $\mathcal{E}_{\mathrm{T}}^{n}$. Then $p_{1} / p_{2}$ is a point of $\mathcal{E}_{\mathrm{T}}^{n}$.

Let us consider $n, p, x, r$. Then $p+\cdot(x, r)$ is a point of $\mathcal{E}_{\mathrm{T}}^{n}$.

Next we state the proposition

(26) For all points $a$, o of $\mathcal{E}_{\mathrm{T}}^{n}$ such that $n \neq 0$ and $a \in \operatorname{Ball}(o, r)$ holds $\left|\sum(a-o)\right|<n \cdot r$.

Let us consider $n$. Note that $\mathcal{E}^{n}$ is real-functions-membered.

One can prove the following propositions:

(27) Let $V$ be an add-associative right zeroed right complementable non empty additive loop structure and $v, u$ be elements of $V$. Then $(v+u)-u=v$.

(28) Let $V$ be an Abelian add-associative right zeroed right complementable non empty additive loop structure and $v, u$ be elements of $V$. Then $(v-$ $u)+u=v$.

(29) For every complex-functions-membered set $Y$ and for every partial function $f$ from $X$ to $Y$ holds $f+c=f+(\operatorname{dom} f \longmapsto c)$.

(30) For every complex-functions-membered set $Y$ and for every partial function $f$ from $X$ to $Y$ holds $f-c=f-(\operatorname{dom} f \longmapsto c)$.

(31) For every complex-functions-membered set $Y$ and for every partial function $f$ from $X$ to $Y$ holds $f \cdot c=f \cdot(\operatorname{dom} f \longmapsto c)$.

(32) For every complex-functions-membered set $Y$ and for every partial function $f$ from $X$ to $Y$ holds $f / c=f /(\operatorname{dom} f \longmapsto c)$.

Let $D$ be a complex-functions-membered set and let $f, g$ be finite sequences of elements of $D$. One can verify the following observations:

* $f+g$ is finite sequence-like,

* $f-g$ is finite sequence-like,

* $f \cdot g$ is finite sequence-like, and

* $f / g$ is finite sequence-like.

Next we state a number of propositions: 
(33) For every function $f$ from $X$ into $\mathcal{E}_{\mathrm{T}}^{n}$ holds $-f$ is a function from $X$ into $\mathcal{E}_{\mathrm{T}}^{n}$.

(34) For every function $f$ from $\mathcal{E}_{\mathrm{T}}^{i}$ into $\mathcal{E}_{\mathrm{T}}^{n}$ holds $f \circ-$ is a function from $\mathcal{E}_{\mathrm{T}}^{i}$ into $\mathcal{E}_{\mathrm{T}}^{n}$.

(35) For every function $f$ from $X$ into $\mathcal{E}_{\mathrm{T}}^{n}$ holds $f+r$ is a function from $X$ into $\mathcal{E}_{\mathrm{T}}^{n}$.

(36) For every function $f$ from $X$ into $\mathcal{E}_{\mathrm{T}}^{n}$ holds $f-r$ is a function from $X$ into $\mathcal{E}_{\mathrm{T}}^{n}$.

(37) For every function $f$ from $X$ into $\mathcal{E}_{\mathrm{T}}^{n}$ holds $f \cdot r$ is a function from $X$ into $\mathcal{E}_{\mathrm{T}}^{n}$.

(38) For every function $f$ from $X$ into $\mathcal{E}_{\mathrm{T}}^{n}$ holds $f / r$ is a function from $X$ into $\mathcal{E}_{\mathrm{T}}^{n}$

(39) For all functions $f, g$ from $X$ into $\mathcal{E}_{\mathrm{T}}^{n}$ holds $f+g$ is a function from $X$ into $\mathcal{E}_{\mathrm{T}}^{n}$.

(40) For all functions $f, g$ from $X$ into $\mathcal{E}_{\mathrm{T}}^{n}$ holds $f-g$ is a function from $X$ into $\mathcal{E}_{\mathrm{T}}^{n}$.

(41) For all functions $f, g$ from $X$ into $\mathcal{E}_{\mathrm{T}}^{n}$ holds $f \cdot g$ is a function from $X$ into $\mathcal{E}_{\mathrm{T}}^{n}$.

(42) For all functions $f, g$ from $X$ into $\mathcal{E}_{\mathrm{T}}^{n}$ holds $f / g$ is a function from $X$ into $\mathcal{E}_{\mathrm{T}}^{n}$.

(43) Let $f$ be a function from $X$ into $\mathcal{E}_{\mathrm{T}}^{n}$ and $g$ be a function from $X$ into $\mathbb{R}^{\mathbf{1}}$. Then $f+g$ is a function from $X$ into $\mathcal{E}_{\mathrm{T}}^{n}$.

(44) Let $f$ be a function from $X$ into $\mathcal{E}_{\mathrm{T}}^{n}$ and $g$ be a function from $X$ into $\mathbb{R}^{1}$. Then $f-g$ is a function from $X$ into $\mathcal{E}_{\mathrm{T}}^{n}$.

(45) Let $f$ be a function from $X$ into $\mathcal{E}_{\mathrm{T}}^{n}$ and $g$ be a function from $X$ into $\mathbb{R}^{1}$. Then $f \cdot g$ is a function from $X$ into $\mathcal{E}_{\mathrm{T}}^{n}$.

(46) Let $f$ be a function from $X$ into $\mathcal{E}_{\mathrm{T}}^{n}$ and $g$ be a function from $X$ into $\mathbb{R}^{\mathbf{1}}$. Then $f / g$ is a function from $X$ into $\mathcal{E}_{\mathrm{T}}^{n}$.

Let $n$ be a natural number, let $T$ be a non empty set, let $R$ be a realmembered set, and let $f$ be a function from $T$ into $R$. The functor $\operatorname{incl}(f, n)$ yields a function from $T$ into $\mathcal{E}_{\mathrm{T}}^{n}$ and is defined by:

(Def. 4) For every element $t$ of $T$ holds $(\operatorname{incl}(f, n))(t)=n \mapsto f(t)$.

We now state several propositions:

(47) Let $R$ be a real-membered set, $f$ be a function from $T$ into $R$, and $t$ be a point of $T$. If $x \in \operatorname{Seg} n$, then $(\operatorname{incl}(f, n))(t)(x)=f(t)$.

(48) For every non empty set $T$ and for every real-membered set $R$ and for every function $f$ from $T$ into $R$ holds $\operatorname{incl}(f, 0)=T \longmapsto 0$.

(49) For every function $f$ from $T$ into $\mathcal{E}_{\mathrm{T}}^{n}$ and for every function $g$ from $T$ into $\mathbb{R}^{1}$ holds $f+g=f+\operatorname{incl}(g, n)$. 
(50) For every function $f$ from $T$ into $\mathcal{E}_{\mathrm{T}}^{n}$ and for every function $g$ from $T$ into $\mathbb{R}^{\mathbf{1}}$ holds $f-g=f-\operatorname{incl}(g, n)$.

(51) For every function $f$ from $T$ into $\mathcal{E}_{\mathrm{T}}^{n}$ and for every function $g$ from $T$ into $\mathbb{R}^{1}$ holds $f \cdot g=f \cdot \operatorname{incl}(g, n)$.

(52) For every function $f$ from $T$ into $\mathcal{E}_{\mathrm{T}}^{n}$ and for every function $g$ from $T$ into $\mathbb{R}^{1}$ holds $f / g=f / \operatorname{incl}(g, n)$.

Let us consider $n$. The functor $\bigotimes_{n}$ yields a function from $\mathcal{E}_{\mathrm{T}}^{n} \times \mathcal{E}_{\mathrm{T}}^{n}$ into $\mathcal{E}_{\mathrm{T}}^{n}$ and is defined by:

(Def. 5) For all points $x, y$ of $\mathcal{E}_{\mathrm{T}}^{n}$ holds $\bigotimes_{n}(x, y)=x y$.

Next we state two propositions:

(53) $\bigotimes_{0}=\mathcal{E}_{\mathrm{T}}^{0} \times \mathcal{E}_{\mathrm{T}}^{0} \longmapsto 0_{\mathcal{E}_{\mathrm{T}}^{0}}$.

(54) For all functions $f, g$ from $T$ into $\mathcal{E}_{\mathrm{T}}^{n}$ holds $f \cdot g=\left(\otimes_{n}\right)^{\circ}(f, g)$.

Let us consider $m, n$. The functor $\operatorname{PROJ}(m, n)$ yields a function from $\mathcal{E}_{\mathrm{T}}^{m}$ into $\mathbb{R}^{\mathbf{1}}$ and is defined as follows:

(Def. 6) For every element $p$ of $\mathcal{E}_{\mathrm{T}}^{m}$ holds $(\operatorname{PROJ}(m, n))(p)=p_{n}$.

One can prove the following propositions:

(55) For every point $p$ of $\mathcal{E}_{\mathrm{T}}^{m}$ such that $n \in \operatorname{dom} p$ holds $(\operatorname{PROJ}(m, n))^{\circ} \operatorname{Ball}(p, r)=$ ]$p_{n}-r, p_{n}+r[$.

(56) For every non zero natural number $m$ and for every function $f$ from $T$ into $\mathbb{R}^{\mathbf{1}}$ holds $f=\operatorname{PROJ}(m, m) \cdot \operatorname{incl}(f, m)$.

\section{Continuity}

Let us consider $T$. One can check that there exists a function from $T$ into $\mathbb{R}^{1}$ which is non-empty and continuous.

Next we state two propositions:

(57) If $n \in \operatorname{Seg} m$, then $\operatorname{PROJ}(m, n)$ is continuous.

(58) If $n \in \operatorname{Seg} m$, then $\operatorname{PROJ}(m, n)$ is open.

Let us consider $n, T$ and let $f$ be a continuous function from $T$ into $\mathbb{R}^{\mathbf{1}}$. Observe that $\operatorname{incl}(f, n)$ is continuous.

Let us consider $n$. One can verify that $\bigotimes_{n}$ is continuous.

One can prove the following proposition

(59) Let $f$ be a function from $\mathcal{E}_{\mathrm{T}}^{m}$ into $\mathcal{E}_{\mathrm{T}}^{n}$. Suppose $f$ is continuous. Then $f \circ-$ is a continuous function from $\mathcal{E}_{\mathrm{T}}^{m}$ into $\mathcal{E}_{\mathrm{T}}^{n}$.

Let us consider $T$ and let $f$ be a continuous function from $T$ into $\mathbb{R}^{1}$. Observe that $-f$ is continuous.

Let us consider $T$ and let $f$ be a non-empty continuous function from $T$ into $\mathbb{R}^{1}$. One can verify that $f^{-1}$ is continuous. 
Let us consider $T$, let $f$ be a continuous function from $T$ into $\mathbb{R}^{\mathbf{1}}$, and let us consider $r$. One can check the following observations:

* $f+r$ is continuous,

* $f-r$ is continuous,

* $f r$ is continuous, and

* $f / r$ is continuous.

Let us consider $T$ and let $f, g$ be continuous functions from $T$ into $\mathbb{R}^{1}$. One can verify the following observations:

$* f+g$ is continuous,

* $f-g$ is continuous, and

* $f g$ is continuous.

Let us consider $T$, let $f$ be a continuous function from $T$ into $\mathbb{R}^{1}$, and let $g$ be a non-empty continuous function from $T$ into $\mathbb{R}^{\mathbf{1}}$. Observe that $f / g$ is continuous.

Let us consider $n, T$ and let $f, g$ be continuous functions from $T$ into $\mathcal{E}_{\mathrm{T}}^{n}$. One can verify the following observations:

$* f+g$ is continuous,

* $f-g$ is continuous, and

* $f \cdot g$ is continuous.

Let us consider $n, T$, let $f$ be a continuous function from $T$ into $\mathcal{E}_{\mathrm{T}}^{n}$, and let $g$ be a continuous function from $T$ into $\mathbb{R}^{\mathbf{1}}$. One can verify the following observations:

* $f+g$ is continuous,

* $f-g$ is continuous, and

* $f \cdot g$ is continuous.

Let us consider $n, T$, let $f$ be a continuous function from $T$ into $\mathcal{E}_{\mathrm{T}}^{n}$, and let $g$ be a non-empty continuous function from $T$ into $\mathbb{R}^{\mathbf{1}}$. Observe that $f / g$ is continuous.

Let us consider $n, T, r$ and let $f$ be a continuous function from $T$ into $\mathcal{E}_{\mathrm{T}}^{n}$. One can verify the following observations:

* $f+r$ is continuous,

* $f-r$ is continuous,

* $f \cdot r$ is continuous, and

* $f / r$ is continuous.

We now state two propositions:

(60) Let $r$ be a non negative real number, $n$ be a non zero natural number, and $p$ be a point of $\operatorname{Tcircle}\left(0_{\mathcal{E}_{\mathrm{T}}^{n}}, r\right)$. Then $-p$ is a point of $\operatorname{Tcircle}\left(0_{\mathcal{E}_{\mathrm{T}}^{n}}, r\right)$. 
(61) Let $r$ be a non negative real number and $f$ be a function from $\operatorname{Tcircle}\left(0_{\mathcal{E}_{\mathrm{T}}^{n+1}}, r\right)$ into $\mathcal{E}_{\mathrm{T}}^{n}$. Then $f \circ-$ is a function from $\operatorname{Tcircle}\left(0_{\mathcal{E}_{\mathrm{T}}^{n+1}}, r\right)$ into $\mathcal{E}_{\mathrm{T}}^{n}$.

Let $n$ be a natural number, let $r$ be a non negative real number, and let $X$ be a subset of $\operatorname{Tcircle}\left(0_{\mathcal{E}_{\mathrm{T}}^{n+1}}, r\right)$. Then $(-) X$ is a subset of $\operatorname{Tcircle}\left(0_{\mathcal{E}_{\mathrm{T}}^{n+1}}, r\right)$.

Let us consider $m$, let $r$ be a non negative real number, and let $X$ be an open subset of $\operatorname{Tcircle}\left(0_{\mathcal{E}_{\mathrm{T}}^{m+1}}, r\right)$. One can verify that $(-) X$ is open.

The following proposition is true

(62) Let $r$ be a non negative real number and $f$ be a continuous function from $\operatorname{Tcircle}\left(0_{\mathcal{E}_{\mathrm{T}}^{m+1}}, r\right)$ into $\mathcal{E}_{\mathrm{T}}^{m}$. Then $f \circ-$ is a continuous function from $\operatorname{Tcircle}\left(0_{\left.\mathcal{E}_{\mathrm{T}}^{m+1}, r\right)}\right.$ into $\mathcal{E}_{\mathrm{T}}^{m}$.

\section{REFERENCES}

[1] Grzegorz Bancerek. Cardinal numbers. Formalized Mathematics, 1(2):377-382, 1990.

[2] Grzegorz Bancerek. The fundamental properties of natural numbers. Formalized Mathematics, 1(1):41-46, 1990.

[3] Grzegorz Bancerek. The ordinal numbers. Formalized Mathematics, 1(1):91-96, 1990.

[4] Grzegorz Bancerek and Krzysztof Hryniewiecki. Segments of natural numbers and finite sequences. Formalized Mathematics, 1(1):107-114, 1990.

[5] Grzegorz Bancerek and Andrzej Trybulec. Miscellaneous facts about functions. Formalized Mathematics, 5(4):485-492, 1996.

[6] Czesław Byliński. The complex numbers. Formalized Mathematics, 1(3):507-513, 1990.

[7] Czesław Byliński. Finite sequences and tuples of elements of a non-empty sets. Formalized Mathematics, 1(3):529-536, 1990.

[8] Czesław Byliński. Functions and their basic properties. Formalized Mathematics, 1(1):5565, 1990.

[9] Czesław Byliński. Functions from a set to a set. Formalized Mathematics, 1(1):153-164, 1990.

[10] Czesław Byliński. Partial functions. Formalized Mathematics, 1(2):357-367, 1990.

[11] Czesław Byliński. Some basic properties of sets. Formalized Mathematics, 1(1):47-53, 1990.

[12] Czesław Byliński. The sum and product of finite sequences of real numbers. Formalized Mathematics, 1(4):661-668, 1990.

[13] Agata Darmochwał. The Euclidean space. Formalized Mathematics, 2(4):599-603, 1991.

[14] Agata Darmochwał and Yatsuka Nakamura. Metric spaces as topological spaces - fundamental concepts. Formalized Mathematics, 2(4):605-608, 1991.

[15] Artur Korniłowicz. Arithmetic operations on functions from sets into functional sets. Formalized Mathematics, 17(1):43-60, 2009, doi:10.2478/v10037-009-0005-y.

[16] Artur Korniłowicz and Yasunari Shidama. Intersections of intervals and balls in $\mathcal{E}_{\mathrm{T}}^{n}$. Formalized Mathematics, 12(3):301-306, 2004.

[17] Artur Korniłowicz and Yasunari Shidama. Some properties of circles on the plane. Formalized Mathematics, 13(1):117-124, 2005.

[18] Beata Padlewska and Agata Darmochwał. Topological spaces and continuous functions. Formalized Mathematics, 1(1):223-230, 1990.

[19] Andrzej Trybulec. Binary operations applied to functions. Formalized Mathematics, 1(2):329-334, 1990.

[20] Andrzej Trybulec. A Borsuk theorem on homotopy types. Formalized Mathematics, 2(4):535-545, 1991.

[21] Andrzej Trybulec. On the sets inhabited by numbers. Formalized Mathematics, 11(4):341$347,2003$.

[22] Andrzej Trybulec and Czesław Byliński. Some properties of real numbers. Formalized Mathematics, 1(3):445-449, 1990. 
[23] Wojciech A. Trybulec. Vectors in real linear space. Formalized Mathematics, 1(2):291-296, 1990.

[24] Zinaida Trybulec. Properties of subsets. Formalized Mathematics, 1(1):67-71, 1990.

[25] Edmund Woronowicz. Relations and their basic properties. Formalized Mathematics, 1(1):73-83, 1990.

[26] Edmund Woronowicz. Relations defined on sets. Formalized Mathematics, 1(1):181-186, 1990.

Received February 9, 2010 